\title{
Laser Propulsion Standardization Issues
}

\author{
Stefan Scharring ${ }^{\mathrm{a}}$, Hans-Albert Eckel ${ }^{\mathrm{a}}$, Hans-Peter Röser ${ }^{\mathrm{b}}$, \\ John E. Sinko ${ }^{\mathrm{c}}$, and Akihiro Sasoh ${ }^{\mathrm{d}}$ \\ ${ }^{a}$ Institute of Technical Physics, German Aerospace Center (DLR), \\ D-70569 Stuttgart, Pfaffenwaldring 38 - 40, Germany \\ ${ }^{b}$ Institute of Space Systems, University of Stuttgart, \\ D-70569 Stuttgart, Pfaffenwaldring 31, Germany \\ ${ }^{c}$ Micro-Nano Global Center of Excellence, Nagoya University (NU), Nagoya, Aichi, Japan T464-8603 \\ ${ }^{d}$ Department of Aerospace Engineering, Nagoya University, Nagoya, Aichi, Japan $\overline{7} 464-8603$
}

\begin{abstract}
It is a relevant issue in the research on laser propulsion that experimental results are treated seriously and that meaningful scientific comparison is possible between groups using different equipment and measurement techniques. However, critical aspects of experimental measurements are sparsely addressed in the literature. In addition, few studies so far have the benefit of independent confirmation by other laser propulsion groups. In this paper, we recommend several approaches towards standardization of published laser propulsion experiments. Such standards are particularly important for the measurement of laser ablation pulse energy, laser spot area, imparted impulse or thrust, and mass removal during ablation. Related examples are presented from experiences of an actual scientific cooperation between NU and DLR. On the basis of a given standardization, researchers may better understand and contribute their findings more clearly in the future, and compare those findings confidently with those already published in the laser propulsion literature. Relevant ISO standards are analyzed, and revised formats are recommended for application to laser propulsion studies.
\end{abstract}

Keywords: Laser ablation, Laser beam characterization, Impulse measurement, Laser propulsion, Standardization

PACS: 06.20.fb, 07.57.Kp, 41.75.Jv, 42.62.-b, 52.38.Mf

\section{INTRODUCTION}

Standardization is a basic issue in experimental work with respect to the comparability of data from different research groups and to possible benefit from international cooperation. In this paper, we present a proposal on standardization issues of the measurement of the impulse coupling coefficient, based on our experiences made within an ongoing collaboration between Nagoya University (NU) and German Aerospace Center (DLR). Parts of this work have recently been discussed in [1]. 


\section{EVIDENT PARAMETERS}

\section{Laser Pulse Energy}

Various energy detectors are used around the world [2], but the most common for high-energy laser beam energy measurements include thermopile-type calorimeters, pyrometers, photodiodes, integrating sphere bolometers, and even wire bolometers [3]. These devices can be wavelength-sensitive. Measurement ranges are usually limited at the upper end by thermoelectric effects, thermal expansion, and damage; and at the lower end by noise and poor background (e.g., due to other light sources in the test area). However, errors in energy measurement typically lie not with the measurement device, but with the interpretation of the operator of optics considerations such as those described above.

In the case of a real-time pulse energy measurement during an ablation experiment, usually a beam-splitter is required. Therefore, a thorough analysis of its ratio of transmission to reflection should be included in the documentation. However, often attenuators are necessary for prevention of damage to the detector, while the laser beam might be focused with a lens or a mirror. For each component, attenuation factors and reflectivity should be mentioned including error bars, if applicable.

The pulse energy might be measured offline with the detector located at the exact position of the target. In most cases, calibrated attenuators are necessary. Furthermore, the laser should be characterized with respect to pulse-to-pulse jitter, yielding an appropriate number of representative shots for offline measurement. In addition, it should be investigated whether the laser system exhibits a baseline drift that contributes to the systematic error.

In general, for the measurement of the laser pulse energy, requirements are set by the manufacturer concerning the illumination of the detector area. Sometimes, it might only be possible to cover a part of the detector area with the laser beam. In that case, special consideration should be taken on spatial variations of the detector sensitivity throughout the detector surface. Especially with locally damaged detectors, large variations can occur. For example, we found a spatial variation of $15.7 \%$ in sensitivity throughout the surface of a damaged Gentec ED-500-LIR detector, while comparable measurements with a calibrated Ophir PE50BB detector yield a variation of 2.9\% [4]. However, once the sensitivity is specified, e.g., by scanning the detector surface with a stable laser probe beam of small diameter, even a damaged detector might be recalibrated for a fixed position of its surface.

\section{Impulse Measurement}

Impulse measurement in laser propulsion was already the subject of intense study; therefore, in this section we will refer to the literature and provide a brief overview.

A thorough study using impulse pendula was carried out by AFRL and DLR $[5,6]$. Torsion pendula have also been addressed; e.g. in [7,8,9]. Piezoelectric force sensors were investigated mainly at UAH; important issues include, e.g., frequency cutoffs, impulse reflection [10], restitution behavior of targets [11], and minimization of noise arising through metal-metal impacts. 
Launches were investigated by many groups $[12,13,14]$. In launch experiments with a parabolic thruster coupling coefficients have been found to tend to be slightly higher than measured at an impulse pendulum [15]. This effect may be attributed to the reduction of degrees of freedom in a pendulum. Only in the case of perfect alignment to the laser beam and an idealized momentum response of the target, the whole kinetic energy will be displayed by the pendulum. Otherwise, kinetic energy may also occur in lateral and / or rotational components. If some of these components are suppressed, e.g., in the case of $2 \mathrm{D}$ motion on an air cushion table, the imparted impulse may be underestimated [16]. However, the whole impulse vector can be reconstructed, e.g., by 3D tracking of a free flight [17].

\section{HIDDEN PARAMETERS}

Standardization efforts with respect to the aforementioned parameters contribute to improve the comparability of experimental data. Nevertheless, a detailed documentation of a laser ablation experiment should include supplementary information. It can be seen from Eq. 1 that for lower fluences the imparted impulse depends on the laser pulse length $\tau[18]$

$$
c_{m} \propto \sqrt[4]{\tau}
$$

while in the plasma regime an explicit dependency on laser wavelength $\lambda$ and intensity $I$ occurs [19]:

$$
c_{m} \propto \frac{1}{\sqrt[4]{I \lambda \sqrt{\tau}}}
$$

Therefore, a sound comparison of impulse coupling data from various experiments should take into account for these parameters; e.g. by plotting $c_{m}$ vs. $I \lambda \sqrt{\tau}[19]$.

\section{Laser Pulse Length}

Comparing the experimental results gathered with the two different laser systems of NU and DLR, we had to take into account for an average difference in the laser pulse length on the order of one magnitude. For the longer pulse, according to Eqs. 1 and 2, this leads to a scaling in the imparted impulse by a factor of 0.75 in the plasma regime and 1.78 at lower fluences. These findings illustrate the importance of the specification of the laser pulse length. The laser pulse length should be denoted as FWHM (full width at half maximum) though other definitions are in use as well; e.g. $10 \%, 5 \%, 1 \%, 1 / \mathrm{e}, 1 / \mathrm{e}^{2}$ of the peak value. Special care has to be taken with the dependence of the pulse length on the pulse energy: For the $\mathrm{CO}_{2}$ high energy laser of DLR we measured an increase of the pulse length (FHWM) by $38 \%$ when enhancing the pulse energy by factor of 5. Furthermore, the pulse shape exhibits a large tail that is especially pronounced with higher pulse energies and might interact with the target like a double pulse system. 


\section{Laser Spot Area}

Eq. 2 demands for the precise knowledge of the spot area when experimental results from different setups are compared. A common way to determine the laser spot area is the employment of thermo-sensitive paper [5]. However, we found that papers from various manufacturers exhibit significant differences with respect to the threshold fluence for coloring and for ablation. Thermo-sensitive paper used at DLR shows coloring for fluences in the range of $0.53 \mathrm{~J} / \mathrm{cm}^{2}$ to $1.53 \mathrm{~J} / \mathrm{cm}^{2}$, while the paper employed by NU already exhibits coloring in the range of $0.32 \mathrm{~J} / \mathrm{cm}^{2}$ to $0.98 \mathrm{~J} / \mathrm{cm}^{2}$ [4]. Exceeding the upper threshold leads to ablation of the paper surface.

Furthermore, even the usage of one type of thermal paper may easily lead to misinterpretations of the spot diameter, since with constant paper sensitivity but increasing laser pulse energy the onset of coloring will drift outwards, depending on the outer slope of the beam profile. On the other hand, this issue provides for a simple method of beam profiling by virtually increasing the paper sensitivity range by a factor $\gamma$ while the laser pulse energy is dimished by $\gamma$, cf. Fig. 1 . However, this approach only works on condition that the normalized beam profile is independent of the pulse energy. In any case, if thermo-sensitive paper is used, its sensitivity should be investigated and specified.

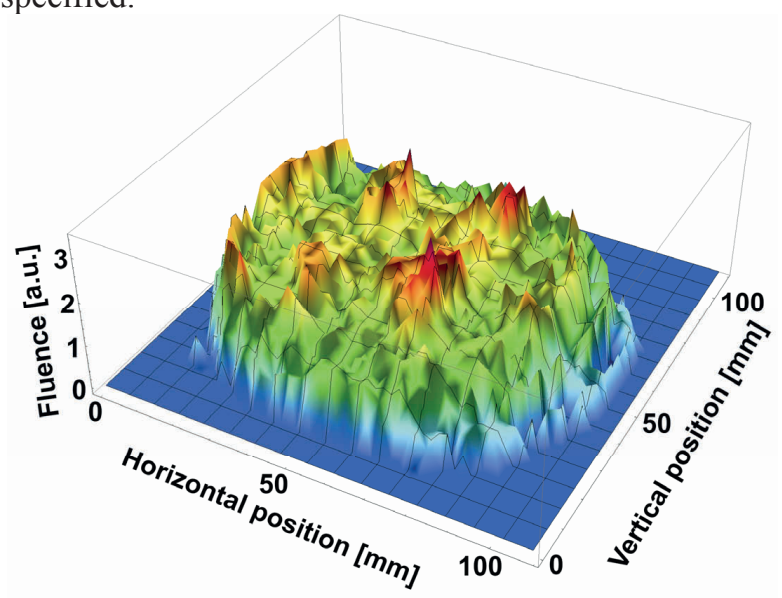

FIGURE 1. Beam profile of the $\mathrm{CO}_{2}$ high energy laser (DLR), generated from burn patterns on thermosensitive paper for 20 different pulse energy levels ranging from around $32 \mathrm{~J}$ to $197 \mathrm{~J}$. For each burn pattern, the moving average over the greyvalue on a field of $3 \times 3$ pixels $(0.38 \times 0.38 \mathrm{~mm})$ was calculated. The fluence was derived on a relative scale by the pulse energy of the sample exhibiting the most pronounced coloring (lowest greyvalue) for the corresponding field.

From a theoretical point of view, the laser spot is defined as the apertured area at which $1-1 / e^{2}$ of the total pulse energy (or power) is measured on a detector behind the aperture, centered on the beam [21]. The accuracy of a measurement according to this method might be limited by the spatial resolution of the corresponding variable aperture and by the pulse-to-pulse stability of the employed laser. In a more elaborate way, the beam diameters $d_{\sigma x}$ and $d_{\sigma y}$ in the directions of the principal axes (transverse to the direction of beam propagation) can be determined according to the second momentum method [22] by: 


$$
\begin{aligned}
& d_{\sigma x}=2 \sqrt{2} \sqrt{\sigma_{x}^{2}+\sigma_{y}^{2}+\gamma \sqrt{\left(\sigma_{x}^{2}-\sigma_{y}^{2}\right)^{2}+4\left(\sigma_{x y}^{2}\right)^{2}}} \\
& d_{\sigma y}=2 \sqrt{2} \sqrt{\sigma_{x}^{2}+\sigma_{y}^{2}-\gamma \sqrt{\left(\sigma_{x}^{2}-\sigma_{y}^{2}\right)^{2}+4\left(\sigma_{x y}^{2}\right)^{2}}}
\end{aligned}
$$

with

$$
\begin{gathered}
\sigma_{x}^{2}=\frac{\iint H(x, y)(x-\bar{x})^{2} d x d y}{\iint H(x, y) d x d y}, \\
\sigma_{y}^{2}=\frac{\iint H(x, y)(y-\bar{y})^{2} d x d y}{\iint H(x, y) d x d y}, \\
\sigma_{x y}^{2}=\frac{\iint H(x, y)(x-\bar{x})(y-\bar{y}) d x d y}{\iint H(x, y) d x d y}, \\
\gamma=\operatorname{sgn}\left(\sigma_{x}^{2}-\sigma_{y}^{2}\right),
\end{gathered}
$$

where $H(x, y)$ denotes the energy distribution. The centroid coordinates $\bar{x}, \bar{y}$ are given by the first order moments:

$$
\begin{gathered}
\bar{x}=\frac{\iint H(x, y) x d x d y}{\iint H(x, y) d x d y}, \\
\bar{y}=\frac{\iint H(x, y) y d x d y}{\iint H(x, y) d x d y} .
\end{gathered}
$$

We analyzed the spot of the DLR laser at a distance of $0.65 \mathrm{~m}$ from the output coupler by scanning with a pyrodetector (Ophir Electronics Ltd., PE50BB). Three attenuation grids were placed in front of the detector. The sensitive area $(46 \mathrm{~mm}$ diameter) was masked by a square aperture $\left(1 \times 1 \mathrm{~cm}^{2}\right)$. Aperture, grids, and detector were fixed relative to each other to account for variations in the spatial response of the detector surface. Discretizing Eqs. 4 and 5, we found beam diameters of $d_{\sigma x}=84.9 \mathrm{~mm}$ and $d_{\sigma y}=83.2 \mathrm{~mm}$ [4], slightly higher than corresponding diameters measured by coloring of thermo-sensitive paper at DLR, $d_{x}=81 \mathrm{~mm}$ and $d_{y}=80 \mathrm{~mm}$.

With respect to ablation, however, it is a practical approach to define the spot area according to the ablation traces. This method can easily be used in a single shot experiment, while a measurement after several ablation events in a series of subsequent laser pulses may lead to misinterpretation of the area size. Surface profilometry may lead to very precise results, but again, great care has to be taken on the exact definition of the ablation crater rim; e.g., by the visible onset of ablation exceeding two times the surface roughness, the position where the $1 / e^{2}$-fraction of the crater maximum depth is located, or the FWHM value of the profile. Redeposition around the crater has to be taken into account, as well [23].

We compared the inner diameter of the ablation spot on POM samples that were taken at different positions of the caustic of a $\mathrm{ZnSe}$ lens with a focal length of $1 \mathrm{~m}$. We defined the ablation area as the inner, glossy part of the spot. The corresponding experimental data are depicted in Table 1. It can be seen that the usage of the thermal 
paper may lead to results ranging from $13 \%$ lower to $31 \%$ higher than the actually ablated spot area, depending on the spot fluence distribution and the area definition for the thermal paper.

TABLE 1. Spot area measurements with thermal paper compared to ablation results with POM.

\begin{tabular}{|c|c|c|c|c|c|}
\hline & \multicolumn{5}{|c|}{ Spot area $\left[\mathrm{cm}^{2}\right]$ (Deviation from POM spot size) } \\
\hline & POM & \multicolumn{4}{|c|}{ Thermal paper } \\
\hline & & \multicolumn{2}{|c|}{$\mathbf{N U}$} & \multicolumn{2}{|c|}{ DLR } \\
\hline & ablated & colored & ablated & colored & ablated \\
\hline$\Phi_{\text {thr }}\left[\mathbf{J} / \mathbf{c m}^{2}\right]$ & $1.35[24]$ & $0.32[4]$ & $0.98[4]$ & $0.53[4]$ & $1.53[4]$ \\
\hline \multicolumn{6}{|l|}{ Position [m] } \\
\hline 1 (Focus) & 1.64 & $2.15(+30.9 \%)$ & $1.92(+17.0 \%)$ & $2.07(+26.2 \%)$ & $1.91(+16.4 \%)$ \\
\hline 1.1 & 2.67 & $3.43(+28.6 \%)$ & $3.19(+19.5 \%)$ & $3.23(+21.2 \%)$ & $3.06(+14.7 \%)$ \\
\hline 1.2 & 4.84 & $5.37(+11.0 \%)$ & $5.07(+4.7 \%)$ & $5.15(+6.4 \%)$ & $4.97(+2.7 \%)$ \\
\hline 1.3 & 8.17 & $9.12(+11.6 \%)$ & $8.65(+5.9 \%)$ & $8.62(+5.6 \%)$ & $8.34(+2.1 \%)$ \\
\hline 1.4 & 14.30 & $13.65(-4.6 \%)$ & $12.97(-9.3 \%)$ & $12.94(-9.5 \%)$ & $12.4(-13.3 \%)$ \\
\hline 1.5 & 18.8 & $18.88(+0.4 \%)$ & $17.78(-5.4 \%)$ & $18.46(-1.8 \%)$ & $17.49(-7.0 \%)$ \\
\hline
\end{tabular}

In the case of a very small focus, the usage of ablation traces or marks on a thermal paper might be impeded by poor spatial resolution. Raytracing software offers an alternative approach by modeling the fluence distribution on the target, e.g. for a cylindrical propellant rod in the focal area of a parabolic mirror [16]. Special care has to be taken with respect to the beam divergence. This may be addressed by modeling with Gaussian beams, though multimode beams might not be covered by the software features. In that case, the beam might be modeled by a customized grid of rays with specifically attributed data describing the distribution of intensity and direction. Hence, beam quality measurements are a necessary prerequisite for this approach.

Diffraction effects on the spot area can also be significant. Often in laser ablation, an aperture is used for beam shaping, for instance to select a part of the beam with a flat intensity profile. Fresnel and / or Fraunhofer diffraction produce a diffraction pattern in an apertured beam profile $[25,26]$. These diffraction effects have a significant influence on the overall area and the fluence distribution of the laser beam spot. Beams may be classified as exhibiting far-field (Fraunhofer region), near-field (Fresnel region), or very-near-field (Geometric optics region) diffractive behavior at a given distance from an aperture. The classification is made using the Fresnel number: $\mathrm{N}_{\mathrm{F}}=A_{a} /(\lambda \mathrm{z})$, where $A_{a}$ is the area of the aperture, $\lambda$ is the laser wavelength, and $\mathrm{z}$ is the distance from the aperture to the target. For $\mathrm{N}_{\mathrm{F}}<\approx 1$, the system is in the far field, and Fraunhofer (first order) diffraction can be used. For $1<\approx \mathrm{N}_{\mathrm{F}}<\approx 100$, Fresnel (second order) diffraction effects dominate. For $\mathrm{N}_{\mathrm{F}}>\approx 100$, geometric optics may be used, and a sharp edge is produced between the aperture and shadow areas.

In most laser propulsion experiments, a flat-top beam was sought; e.g., in order to identify fluence thresholds. This most closely corresponds to the geometric optics case. For producing a uniform beam shape, placement of the aperture directly atop the target may be necessary, but interaction effects with the aperture may then influence the ablation process. For a remote-type laser propulsion system, Fraunhofer diffraction should be dominant. Due to the implications for real systems, it is probably a good idea to state the operational regime of validity (i.e., Fraunhofer, Fresnel, or geometric) when reporting laboratory laser ablation propulsion experiments. 
Finally, reports on laser ablation experiments should supply detailed information on the surrounding geometry. The confining nozzle should be specified with respect to the expansion ratio of ablated area to aperture area [10], wherever possible. Even ablation craters that grow during a laser burst represent a confinement for the ablation event of the subsequent pulses [26]. Hence, the number of pulses on a target and at least the overall ablated mass should be specified as well. Special consideration has to be given to the focusing optics: It should be noted whether the laser beam is externally focused onto the target surface or whether the nozzle itself is part of the focusing optics. In the latter case, impulse enhancement may occur by an absorption wave that is raised on the reflected segment of the laser rays [4].

\section{CONCLUSIONS}

Although a wide range of measurement techniques is used throughout the world, it is the hope of the authors that some significant agreement may be reached about standards for measurements to produce comparable experiments and increase the quality of research. Such standards should be based on direct measurement parameters of maximal importance, including the laser pulse energy, pulse length, spot area, imparted impulse, and ablated mass.

\section{ACKNOWLEDGMENTS}

Financial support by DLR funds for International Cooperation is gratefully acknowledged, and additional support was provided through the Dr. John Sinko by a 2009 Global Center of Excellence Young Researcher Award. Support was also provided by Nagoya University and the Micro-Nano Division of the Global Center of Excellence.

\section{REFERENCES}

1. J. E. Sinko, S. Scharring, H.-A. Eckel, H.-P. Röser, and A. Sasoh, "Measurement Issues In Pulsed Laser Propulsion", in $6^{\text {th }}$ International Symposium on Beamed Energy Propulsion, 2009, edited by C.R. Phipps, K. Komurasaki, and J.E. Sinko, AIP Conference Proceedings, American Institute of Physics, Melville, NY, to be published.

2. H. L. Hackforth, Infrared Radiation, New York: McGraw-Hill Book Company, Inc., 1960, $1^{\text {st }}$ edition, pp. 142-187.

3. Yu. A. Rezunkov, A. L. Safronov, A. A. Ageichik, M. S. Egorov, V. V. Stepanov, V. S. Rachuk, V. Yu. Guterman, A. V. Ivanov, S. G. Rebrov, and A. N. Golikov, "Performance Characteristics of Laser Propulsion Engine Operating both in CW and in Repetitively-Pulsed Modes", in $4^{\text {th }}$ International Symposium on Beamed Energy Propulsion, 2005, edited by K. Komurasaki, T. Yabe, S. Uchida, and A. Sasoh, AIP Conference Proceedings 830, American Institute of Physics, Melville, NY, 2006, pp. 3-13.

4. S. Scharring, J. Sinko, A. Sasoh, H.-A. Eckel, H.-P. Röser, "Experimental Determination of the Impulse Coupling Coefficient - Standardization Issues", Int. J. Aerospace Innovations, submitted for publication.

5. W. O. Schall, W. L. Bohn, H.-A. Eckel, W. Mayerhofer, W. Riede, S. Walther, and E. Zeyfang, "US German Lightcraft Impulse Measurements", EOARD Grant F61775-00-WE033, Final report (2001)

6. W. O. Schall, H.-A. Eckel, W. Mayerhofer, W. Riede, and E. Zeyfang, "Comparative lightcraft impulse measurements", in High-Power Laser Ablation IV, 2002, edited by C.R. Phipps, Proc. of SPIE 4760, 2002, pp. 908-917. 
7. K. Suzuki, K. Sawada, R. Takaya, and A. Sasoh, "Ablative Impulse Characteristics of Polyacetal with Repetitive $\mathrm{CO}_{2}$ Laser Pulses", Journal of Propulsion and Power 24(4), 834-841 (2008).

8. C. R. Phipps, J. R. Luke, W. Helgeson, and R. Johnson, "A ns-Pulse Laser Microthruster", in $4^{\text {th }}$ International Symposium on Beamed Energy Propulsion, 2005, edited by K. Komurasaki, T. Yabe, S. Uchida, and A. Sasoh, AIP Conference Proceedings 830, American Institute of Physics, Melville, NY, 2006, pp. 235-246.

9. B. C. D'Souza and A. D. Ketsdever, "Direct Impulse Measurements of Ablation Processes from Laser-Surface Interactions", in $36^{\text {th }}$ AIAA Plasmadynamics and Lasers Conference, AIAA Paper 2005-5172.

10.E. Sterling, J. Lin, J. Sinko, L. Kodgis, S. Porter, A. V. Pakhomov, C. W. Larson, and F. B. Mead, Jr., "Laser-Driven Mini-Thrusters", in $4^{\text {th }}$ International Symposium on Beamed Energy Propulsion, 2005, edited by K. Komurasaki, T. Yabe, S. Uchida, and A. Sasoh, AIP Conference Proceedings 830, American Institute of Physics, Melville, NY, 2006, pp. 247-258.

11.J. E. Sinko, and J. S. Lassiter, "Sphere-Wall Impact Experiments with Piezoelectric Force Sensors", in $5^{\text {th }}$ International Symposium on Beamed Energy Propulsion, 2007, edited by A. V. Pakhomov, AIP Conference Proceedings 997, American Institute of Physics, Melville, NY, 2008, pp. 131-142.

12. M. M. Michaelis, A. Moorgawa, A. Forbes, W. Klopper, E. McKenzie, D. Boutchiama, and H. Bencherif, "Laser Propulsion Experiments in South Africa", in High-Power Laser Ablation IV, 2002, edited by C.R. Phipps, Proc. of SPIE 4760, 2002, pp. 691-695.

13. K. Watanabe, T. Takahashi, and A. Sasoh, "Useful In-space Impulse Generation Powered by Laser Energy", in $2^{\text {nd }}$ International Symposium on Beamed Energy Propulsion, 2003, edited by K. Komurasaki, AIP Conference Proceedings 702, American Institute of Physics, Melville, NY, 2004, pp. 115-121.

14. L. N. Myrabo, "Brief History of the Lightcraft Technology Demonstrator (LTD) Project", in $I^{s t}$ International Symposium on Beamed Energy Propulsion, 2002, edited by A. V. Pakhomov, AIP Conference Proceedings 664, American Institute of Physics, Melville, NY, 2003, pp. 49-60.

15.S. Scharring, H.-A. Eckel und H.-P. Röser, "Flight Analysis of a Parabolic Lightcraft", in $5^{\text {th }}$ International Symposium on Beamed Energy Propulsion, 2007, edited by A. V.Pakhomov, AIP Conference Proceedings 997, American Institute of Physics, Melville, NY, 2008, pp. 304-315.

16. S. Scharring, E. Wollenhaupt, H.-A. Eckel und H.-P. Röser, "Flight Experiments on Energy Scaling for In-Space Laser Propulsion", in $6^{\text {th }}$ International Symposium on Beamed Energy Propulsion, 2009, edited by C. R. Phipps, K. Komurasaki, and J. E. Sinko, AIP Conference Proceedings, American Institute of Physics, Melville, NY, to be published.

17. S. Scharring, H.-A. Eckel und H.-P. Röser, "High speed Analysis of Free Flights with a Parabolic Thruster", in $6^{\text {th }}$ International Symposium on Beamed Energy Propulsion, 2009, edited by C. R. Phipps, K. Komurasaki, and J. E. Sinko, AIP Conference Proceedings, American Institute of Physics, Melville, NY, to be published.

18. C. Phipps., Jr., T. Turner, R. Harrison, G. York, W. Osborne, G. Anderson, X. Corlis, L. Haynes, H. Steel, K. Spicochi, and T. King, "Impulse Coupling to Targets in Vacuum by KrF, HF and CO2Lasers", J. Appl. Phys. 64, 1083-1096 (1988).

19. J. E. Sinko and D. A. Gregory, "Models for Laser Ablation Mass Removal and Impulse Generation in Vacuum", in $6^{\text {th }}$ International Symposium on Beamed Energy Propulsion, 2009, edited by C. R. Phipps, K. Komurasaki, and J. E. Sinko, AIP Conference Proceedings, American Institute of Physics, Melville, NY, to be published.

20.C. Phipps, M. Birkan, W. Bohn, H.-A. Eckel, H. Horisawa, Th. Lippert, M. Michaelis, Yu. Rezunkov, A. Sasoh, W. Schall, S. Scharring, J.E. Sinko, "Laser Ablation Propulsion", J. of Propulsion and Power, to be published.

21."Lasers and laser-related equipment - Test methods for laser beam widths, divergence angles and beam propagation ratios - Part 3: Intrinsic and geometrical laser beam classification, propagation and details of test methods", ISO 11146-3:2004(E), ISO copyright office, Geneva (2004).

22. "Lasers and laser-related equipment - Test methods for laser beam widths, divergence angles and beam propagation ratios - Part 1: Stigmatic and simple astigmatic beams", ISO 111461:2005(E), ISO copyright office, Geneva, 2005

23. J. E. Sinko, S. Scharring, H.-A. Eckel, Y. Tsukiyama, K. Ichihashi, N. Ogita, A. Sasoh, N. Umehara, H.-P. Röser, " $\mathrm{CO}_{2}$ Laser Ablation Area Scaling and Redeposition with Flat Polyoxymethylene Targets" (these proceedings, submitted for publication) 
24.J. Sinko, "Vaporization and shock wave dynamics for impulse generation in laser propulsion", Ph.D. Thesis, The University of Alabama in Huntsville, 2008

25. M. Born and E. Wolf, Principles of Optics, Cambridge University Press, $7^{\text {th }}$ ed., 1999, pp. $412-446$.

26.K. D. Mielenz, "Algorithms for Fresnel Diffraction at Rectangular and Circular Apertures", J. Res. Natl. Stand. Technol. 103(5), 497-509 (1998).

27.H. Maesato, E. Koizumi, and H. Tahara, "Performance Characteristics of Low-Power Laser Ablative Thrusters for Small Satellites", in $4^{\text {th }}$ International Symposium on Beamed Energy Propulsion, 2005, edited by K. Komurasaki, T. Yabe, S. Uchida, and A. Sasoh, AIP Conference Proceedings 830, American Institute of Physics, Melville, NY, 2006, pp. 284-294. 